\title{
DEVELOPMENT OF LEARNING ACTIVITY STATUS RECORDING SYSTEM USING THE INTERNET OF THINGS
}

\author{
Atsushi Iwayama ${ }^{1}, \&$ Yosuke Ito ${ }^{2}$ \\ ${ }^{1}$ Hyogo University of Education (Japan) \\ ${ }^{2}$ Naruto University of Education (Japan)
}

\begin{abstract}
In countries with birth rates that are declining significantly such as Japan, integration and abolition of schools have recently been attempted over a wide area to maintain a standard school scale from an educational point of view. Therefore, it has become difficult to carry out educational activities across different schools with the full cooperation of teachers. The task of educating must be performed without disparity, regardless of area, by complementing teachers' educational knowledge and skills. One solution is to realize an advanced education support method by promoting distance education. The aim of this study is to develop a system to record learning activity status to support classes from a distance using the Internet and the Internet of Things (IoT). The educational support system consists of a video conference system, online storage service, 'learning activity status recording system (LRS)', and teaching materials with IoT. This paper introduces LRS and describes how to transmit state transition information from a wheel mobile robot as a teaching material with IoT to LRS. From the results of surveys on the developed educational support system for high school teachers, the usefulness of the system was clarified.
\end{abstract}

Keywords: Distance education, learning activity, robot, IoT.

\section{Introduction}

In countries with birth rates that are declining significantly such as Japan, integration and abolition of schools have recently been attempted over a wide area to maintain a standard school scale from an educational point of view. However, there are small schools that cannot be administratively integrated, but such schools are required to have the same high-quality education as large schools. In terms of future education in Japan, the Ministry of Education, Culture, Sports, Science and Technology (MEXT) has set a goal to realize high-quality education for all students through the use of advanced technology. The realization of advanced education through the promotion of distance education is listed as one of its goals. One example of distance education that has been reported is the lecture. Most cases of the application of distance education to classes, including experiments and practical training, have not been reported.

The aim of this study was to develop a system to record learning activity status to support classes from a distance using the Internet and the Internet of Things (IoT). Japanese junior high school students learn Japanese, mathematics, science, society, foreign languages, music, art, health and physical education, technology, and home economics. In the domain of technology, improvement of education on programming is required as part of the recent educational policy. In past research, we proposed a support method from a remote location of classes targeted learning contents 'measurement and control by program' stipulated in the course of study the junior high school and developed the system (Iwayama \& Ito, 2017). The educational support system consists of a video conference system (VCS), online storage service, 'learning activity status recording system (LRS)', and teaching materials with IoT. This paper introduces LRS and describes how to transmit state transition information from a wheel mobile robot as a teaching material with IoT to LRS.

\section{Distance educational support system}

In many schools, it is often difficult to conduct lessons on the same content at the same time. Therefore, this system has functions that can support education asynchronously. The educational support system employed in distance schools uses an online storage service, so that data can be stored and moved between servers set up in schools via the Internet (Figure 1). LRS is installed in each school, and the faculty is configured to transmit and receive the data through the online storage service, as necessary. The 
teacher of school A is the leader. Other teachers of schools Bi, $\mathrm{i}=1 \sim \mathrm{n}$ work in cooperation with the leader. The leader is assumed to have higher skills than the other teachers. The leader performs lectures for all students using VCS, as usual. Teachers in each school Bi are involved in virtual team teaching and simultaneously conduct classes for the corresponding students. The students can ask questions of the teacher in the school to which they belong. If the teacher of school Bi cannot respond to a specific question, the leader teacher of school A answers the students, instead.

Each learning material is configured for IoT. The teaching material is a wheel mobile robot, with a wireless LAN module and sensors to measure the rotation of the wheel inside. The state transition information of the teaching material is sent to LRS via a wireless LAN in the classroom, where the lesson is conducted by IoT. State transition information of teaching materials aggregated during practical training is converted into coordinate data in the server by the developed conversion software. The converted coordinate data are stored in a database with permission to be able to refer by the web service program. The saved data can be transmitted to teachers in each school through online storage. Therefore, since all teachers can grasp the state transition of each teaching material, teachers of school A and teachers of $\mathrm{Bi}$ school can cooperate with one another. As a result, the teachers in school Bi can teach their students while assisting from the teacher in school A.

Figure 1. Distance educational support system.

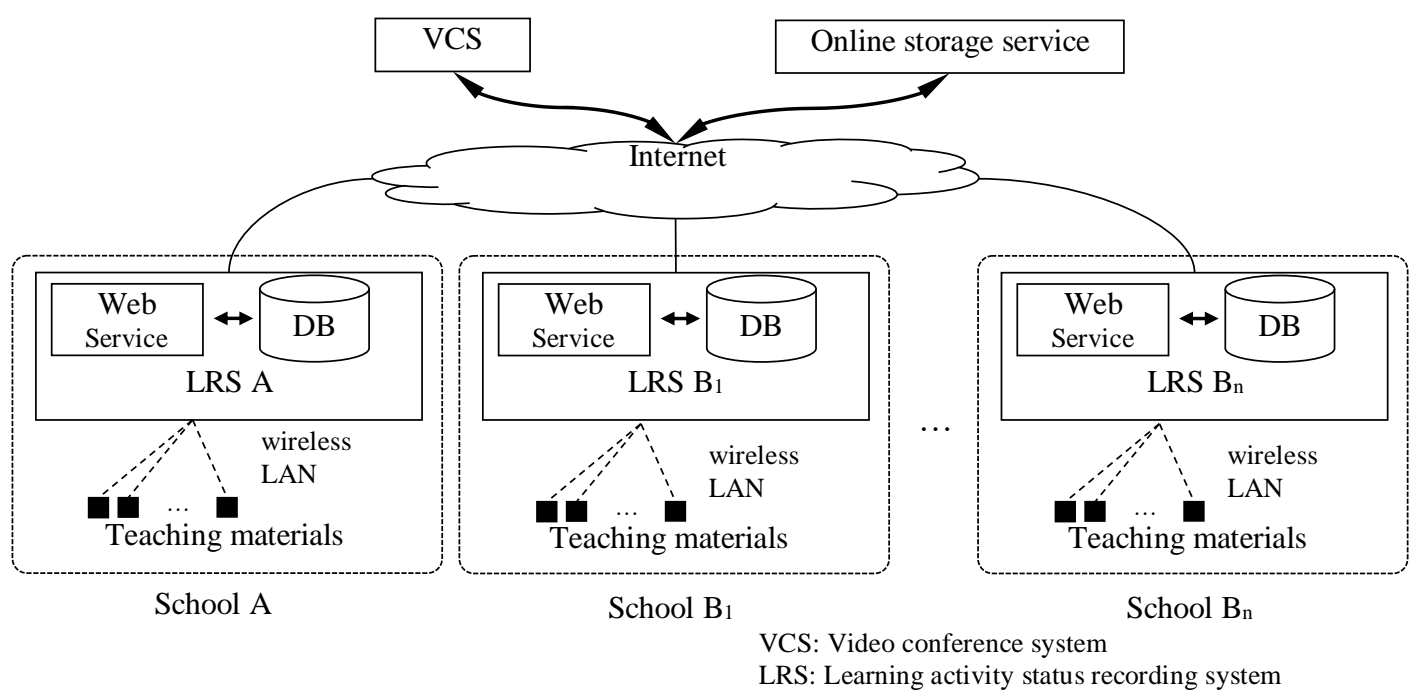

\section{Learning activity status recording system}

The learning activity status recording system saves the activity situation and displays the trajectory based on the analysis, in the distance educational support system. An outline of the configuration is shown in Figure 2. The OS of this system is Linux, and the database uses MySQL. The state transition information transmitted from the teaching material with IoT consists of identification code, time, and rotation counts for both side wheels. Message Queue Telemetry Transport (MQTT) protocol is adopted because it is programming language-independent and lightweight for network traffic (Hwang, Park, \& Shon, 2016). MQTT is a messaging protocol based on the TCP / IP stack. It is open-source and free of charge. Libraries for MQTT use are provided for various programming languages. The MQTT message communication method involves transmission from a Publisher (the calling side) and reception by a Subscriber (the receiving side) via a server called the MQTT Broker. Mosquitto, an MQTT Broker provided as open-source software, is employed. A library for Python, a dynamic programming language used in various applications, is adapted to store data sent from the Publisher to the Subscriber in the database.

The software embedded in the teaching material is in the process of development. Transmission experiments are performed using Linux (Raspberry Pi), instead of the teaching material. A script written by Python sends the state transition information to the database using MQTT at specific intervals. The data are sent to the database in DB1 illustrated in Figure 2. They are converted to two-dimensional coordinates by the developed program. Odometry is algorithm for estimating the position of a robot by computing the displacement vector based on rotation speed and angle of the right and left side wheels in a wheel-moving robot. The developed program is described using odometry. The transformed coordinate values are stored in DB2 illustrated in Figure 2. Web contents for visually providing the information 
necessary for educational support are constructed using Hypertext Preprocessor (PHP), which is an open-source general script language. It is configured to be delivered from the web server after forming a vector image with JavaScript to improve design ability. The teachers and students can access the database via web contents and confirm the trajectory connecting points of coordinate values with lines.

Figure 2. Constitution of LRS.

Linux

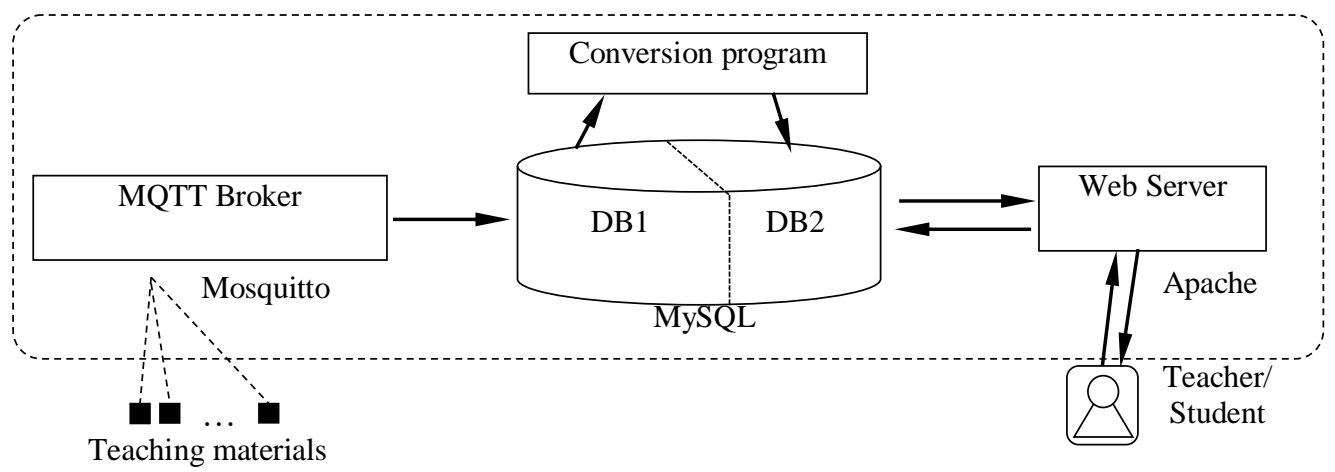

\section{Awareness survey of the system}

Twenty-five teachers in charge of technology education were interviewed following an explanatory overview of the distance educational support system. The content of the survey confirms the usefulness of the system from an educational point of view (Table 1). Positive opinions were found in all items as a result of the survey.

Table 1. Evaluation of educational support system $(n=25)$.

\begin{tabular}{|l|c|c|c|}
\hline \multicolumn{1}{|c|}{ Question } & Positive response (\%) & Average & S. D. \\
\hline $\begin{array}{l}\text { Do you think that the educational support system from the } \\
\text { remote place of the class including the practical training which } \\
\text { received explanation this time is useful? }\end{array}$ & 92.0 & 3.24 & 0.71 \\
\hline $\begin{array}{l}\text { Do you think that classes can be developed while listening to } \\
\text { the opinions of experts on a system for educational support } \\
\text { from remote areas? }\end{array}$ & 88.0 & 3.32 \\
\hline $\begin{array}{l}\text { Do you think teachers can perceive learners' thinking and } \\
\text { ingenuity, even in remote schools, by using an educational } \\
\text { support system in remote areas? }\end{array}$ & 92.0 & 0.68 \\
\hline $\begin{array}{l}\text { Do you think that it is worthwhile, not only in the technical } \\
\text { field but also for any subject? }\end{array}$ & 88.0 & 3.20 & 0.69 \\
\hline
\end{tabular}

\section{Conclusions}

The state of teaching materials with IoT can be visualized by the developed conversion software. It was shown that students and teachers in remote locations could view the learning process of the other students and the learning outcome, which is the trajectory of the robot, using a web browser. The results of interviews with teachers of technical education implied its usefulness. In the future, we will conduct an examination that actually receives the state transition information from the robot and compare the mobile situation and trajectory of the web contents.

\section{References}

Ben-Ari, M., \& Mondada, F. (2017). Robotic Motion and Odometry. Elements of Robotics, pp. 63-93.

Hwang, H. C., Park, J., \& Shon, J. G. (2016). Design and implementation of a reliable message transmission system based on MQTT protocol in IoT. Wireless Personal Communications, Vol. 91, Issue 4, pp. 1765-1777.

Iwayama, A., \& Ito, Y. (2017). A distance educational support method for class with practical training using IoT. Technology Education New Zealand (TENZ) Conference and International Conference on Technology Education, pp. 82-89. 